\title{
Efectividad de la Expansión Maxilar en la Corrección de Apiñamiento en Dentición Mixta. Revisión Sistemática
}

\author{
Effectiveness of the Maxillary Expansion in the Correction \\ of Crowding and Mixed Dentition. Systematic Review
}

\author{
María Carolina Longlax Triana'; Gustavo Jaimes Monroy ${ }^{2,3}$; \\ Nidia Jackeline Boada Cuesta² \& Adriana Marcela Lugo Latorre ${ }^{2}$
}

LONGLAX, T. M. C.; MONROY, G. J.; BOADA, C. N. J. \& LUGO, L. A. M. Efectividad de la expansión maxilar en la corrección de apiñamiento en dentición mixta. Revisión Sistemática. Int. J. Odontostomat., 14(1):101-108, 2020.

RESUMEN: El apiñamiento en edades tempranas, se refleja cuando el tamaño dental no coincide con la longitud de arco. De este modo, resulta importante determinar si la expansión del maxilar podría solucionar por sí solo el problema de apiñamiento en el sector anterior, teniendo en cuenta que este tratamiento es uno de los más antiguos y que comúnmente se ha enfocado en la resolución de alteraciones transversales en dientes posteriores empleándose a edades tempranas. El objetivo fue determinar si la expansión maxilar es efectiva o no, en la corrección del apiñamiento en dentición mixta mediante el análisis de la evidencia disponible. Se realizó una revisión sistemática en español e inglés, para establecer la máxima evidencia posible, con las indicaciones de la guía PRISMA. Dos artículos cumplieron con la calidad de la revisión. En el primero se estableció que el apiñamiento mejoro despues del tratamiento de expansion maxilar, con una recidiva menor en la dentición permanente. En el segundo, se encontró que el apiñamiento en los incisivos permanentes superiores fue corregido cuando se retiró el aparato expansor. Se concluyó que la evidencia actual no tiene la suficiente calidad metodológica para llevar a una toma de decisión. Se recomienda hacer ensayos clínicos aleatorizados en este tema.

PALABRAS CLAVE: dentición mixta, ortodoncia preventiva, maloclusión, revisión (DeCS).

\section{INTRODUCCIÓN}

La maloclusión se define como una oclusión anormal en la que los dientes no están en una posición adecuada en relación con los dientes adyacentes del mismo maxilar, o con los dientes opuestos cuando los maxilares están en cierre (Mafla, 2011; Howe et al., 1983). Entre las manifestaciones más notables de las maloclusiones se encuentra el apiñamiento dental, el cual, se define como la diferencia en la relación entre el tamaño dental y el tamaño del maxilar, que conduce a la rotación de los dientes (Warren, 2003; Lombardi, 1982).

Además de ser considerada como la más común de las maloclusiones, existe una discusión sobre su origen, algunos investigadores (Arif et al., 2014; van der Linden, 2008) consideran que el apiñamiento se puede verificar según la discrepancia entre la longitud de arco disponible y la suma de los diámetros mesodistales de los dientes, determinados principalmente por los factores genéticos; otros autores, refieren que el tamaño del arco, sobre todo en anchura, está íntimamente relacionado con el grado de apiñamiento (Normando et al., 2013; McKeown, 1981; Radnzic, 1988). Adicionalmente, también existe la posición de que hay una relación entre el tamaño de los dientes y el apiñamiento que determina su presencia (Lundstrom, 1951; Doris et al., 1981).

Estas diferentes propuestas, sobre la etiología, enfocadas en relacionarlo con el tamaño del arco en anchura, o al tamaño de los dientes, o a la combinación de las dos anteriores (Barber Starr, 1999), crean

\footnotetext{
${ }^{1}$ Directora del Postgrado de Ortodoncia Universidad Antonio Nariño. Facultad de Odontología. Bogotá D.C. Colombia.

2 Postgrado de Ortodoncia de la Universidad Antonio Nariño. Facultad de Odontología. Grupo de Investigación en Salud Oral. Bogotá D.C. Colombia.

${ }^{3}$ Docente investigador de la Escuelas de Ciencias de la Salud-ECISA. Universidad Nacional Abierta y a Distancia-UNAD. Bogotá D.C. Colombia.
} 
entre los clínicos controversia, en lo que se refiere, a cuál de estas teorías es la más acertada y del mismo modo cual es la forma más efectiva de tratar el apiñamiento en dentición mixta (Baccetti et al., 2008; Lione et al., 2013) . Es así, como se instauran o no, tratamientos en esta dentición; algunos enfocados al tamaño dental, otros al tamaño del maxilar en sentido transversal y finalmente, un grupo de especialistas que consideran no realizan tratamiento temprano (Sunnak et al., 2015; Vaughn, 2005) y prefieren esperar a una dentición permanente y dar solución con aparatología fija ortodóntica y posibles exodoncias (King \& Brudvik, 2010; Kau 2004; Canuto et al., 2010). Estas diferencias tan grandes pueden estar asociadas a la falta de evidencia científica, que permita la toma de decisiones terapéuticas adecuadas.

Un tratamiento muy reconocido es la expansión de los arcos dentales mediante la incorporación de aparatos fijos o removibles (Graber Thomas, 2006). La expansión maxilar, se utiliza principalmente para corregir las discrepancias transversales del maxilar superior (Tang, 2015), pero otras indicaciones de uso incluyen el aumento del perímetro del arco para aliviar el apiñamiento dental moderado, discrepancias de la longitud del tamaño del arco y facilitar el tratamiento sin exodoncias (Marshall et al., 2008; Cozzani et al., 2007). De este modo, la expansión maxilar se muestra como una alternativa de tratamiento para corregir el apiñamiento dental en dentición mixta.

Estudios realizados a partir del año 2006, sobre la estabilidad a largo plazo de la expansión maxilar sólo han proporcionado información adicional limitada sobre la base de evidencia débil (Bartzela \& Jonas, 2007). En la actualidad existe controversia sobre la efectividad de la expansión maxilar en la corrección de apiñamiento en dentición mixta. El objetivo de esta revisión sistemática, con la evidencia disponible, es determinar si la expansión maxilar es efectiva para corregir el apiñamiento en dentición mixta.

\section{MATERIAL Y MÉTODO}

Esta revisión sistemática se realizó bajo la estructura de Preferred Reporting Items for Systematic Reviews and Meta-Analysis - PRISMA (Johansen \& Thomsen, 2016; Liberati et al., 2009). Se utilizó el formato PICO (Bragge, 2010) para las revisiones sistemáticas (Tabla I).

Dos investigadores de forma independiente realizaron la búsqueda en las bases de datos electrónicas, PubMed, ScienceDirect, EBSCO, Google Académico y en el metabuscador Trip Database.

Para la selección de los artículos, se tuvieron en cuenta, los siguientes criterios de inclusión: estudios en humanos, pacientes en crecimiento con dentición mixta, artículos en inglés y español, ensayos clínicos aleatorizados, metaanálisis, estudios prospectivos, estudios retrospectivos; producidos desde 2006 a 2016, que no indicaran el uso de aparatología previa, y que refirieran estabilidad mayor o igual a dos años. Los criterios de exclusión fueron: Artículos con reportes de caso, series de casos, artículos en otros idiomas, pacientes con síndromes de labio y paladar fisurado, pacientes con anomalías dentales y faciales, estudios en adultos, estudios en pacientes quirúrgicos, estudios en dos fases.

Para la búsqueda electrónica, se utilizaron tres algoritmos en español e inglés:

1. Español: "Expansión Maxilar" AND Apiñamiento AND Corrección AND "Dentición Mixta".

Inglés: "Maxillary Expansion" AND Crowding AND Correction AND "Dentition Mixed"

2. Español: "Expansión Maxilar" OR "Expansión Palatina" AND Apiñamiento OR Maloclusión AND Corrección OR Resolución AND "Dentición Mixta" OR "Dentición Transitoria"

Inglés: "Maxillary Expansion" OR "Palatal Expansion Technique" AND Crowding OR Malocclusion AND Correction OR Resolution AND "Dentition Mixed" OR "Dentition Transitional"

3. Español: "Expansión Maxilar" OR "Expansión palatina" AND Apiñamiento OR Maloclusión Inglés: "Maxillary Expansion" OR "Palatal Expansion Technique" AND Crowding OR Malocclusion.

Se obtuvieron todos los artículos relacionados con las palabras de búsqueda. Se registraron los resultados y se tamizaron inicialmente por títulos y resúmenes. Se eliminaron de esta manera, todas las publicaciones duplicadas, las que carecían de información clara o que se verificaba poco rigor metodológico o baja relación con el objetivo de la revisión. Los resultados de cada investigador se registraron en Excel 2013 y luego se compararon los resultados de cada uno. Las discrepancias 


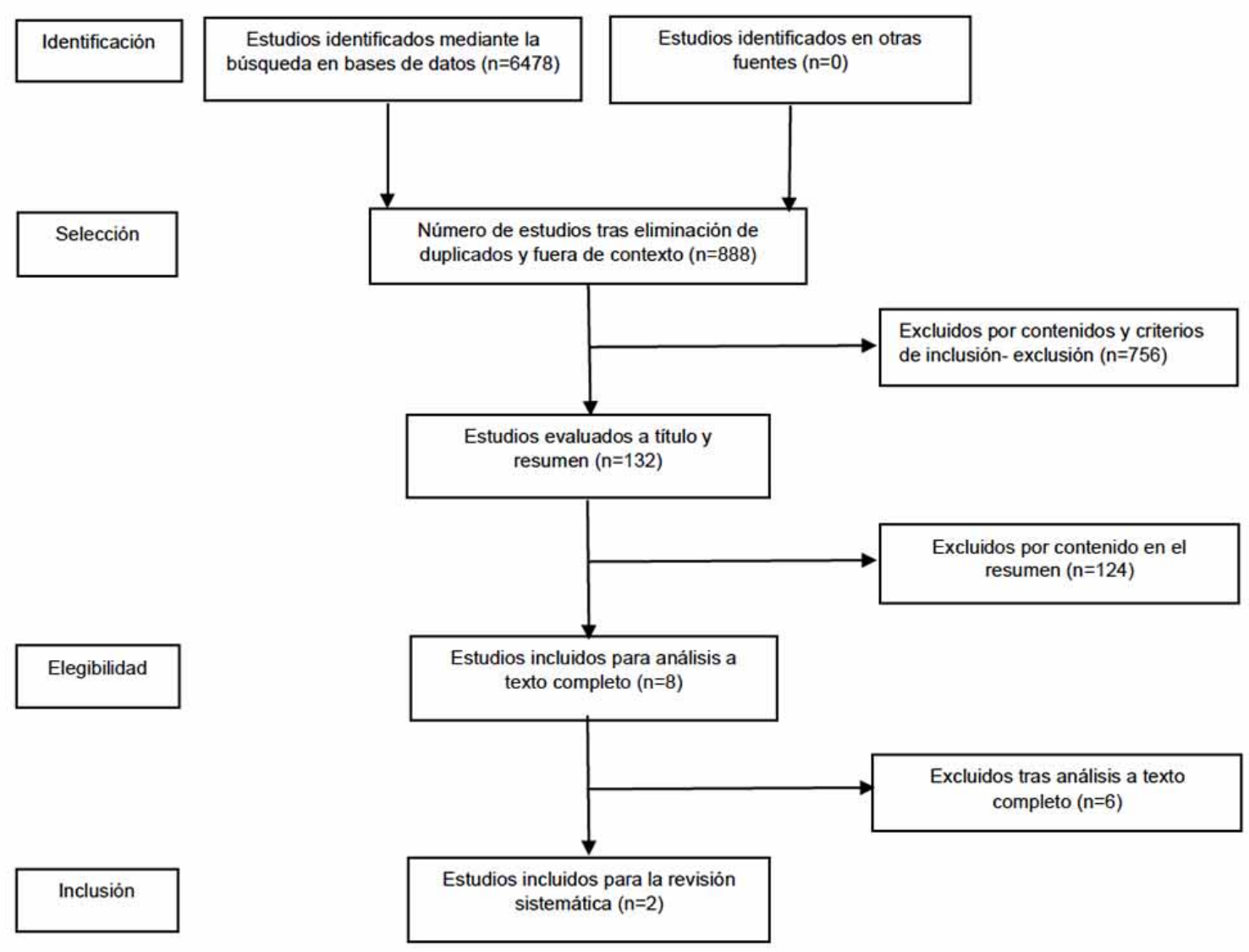

Fig. 1. Esquema de tamizaje de los artículos estructura Prisma.

Tabla I. Formato de pregunta PICO sobre expansión maxilar.

\begin{tabular}{ll} 
Participantes & Pacientes en dentición mixta \\
\hline Intervención & Expansión maxilar \\
Comparación & Grupo œntrol no intervenido \\
Outcome & Efectividad o no \\
Hipótesis nula & Se corrige el apiñamiento en dentición mixta con expansión maxilar \\
\hline
\end{tabular}

se aclararon por discusión grupal y acuerdo entre los autores.

A continuación, siguiendo los criterios de inclusión y exclusión, se seleccionaron todos los artículos que relacionaron expansión maxilar con corrección de apiñamiento de manera precisa en concordancia con el objetivo de la revisión. Una vez efectuada esta etapa, se obtuvieron los artículos a texto completo y dos evaluadores realizaron la calificación metodológica de manera independientemente, tomando como guía la Revisión Sistemática efectuada por Lagraver en 2005, (Tabla IV). Los puntajes de esta escala se distribuyen de acuerdo con la calidad que se cumple al verificar sus componentes: de 1 a 7 puntos significa una publicación con las mayores carencias y se cataloga como de baja calidad; desde 8 hasta 13 puntos se considera de calidad moderada, y de 14 a 20 puntos la publicación se considera de alta calidad (Lagravere et al., 2005). Para valorar el acuerdo entre los calificadores se realizó un cálculo del coeficiente de correlación intraclase mediante el programa libre Real Statistics con valores que resultaron en alta concordancia de 0,88 (Trevethan, 2017; Koo \& Li, 2016).

Análisis e integración de la información. Los resultados de la búsqueda se observan en la Figura 1, 
LONGLAX, T. M. C.; MONROY, G. J.; BOADA, C. N. J. \& LUGO, L. A. M. Efectividad de la expansión maxilar en la corrección de apiñamiento en dentición mixta. Revisión Sistemática. Int. J. Odontostomat., 14(1):101-108, 2020

en el diagrama de flujo con la estructura PRISMA. La búsqueda electrónica arrojo como resultado 6.478 artículos, de los cuales 5.590 fueron registros o citas duplicadas o no eran relacionados con el tema lo que dio como información efectiva 888 artículos: PubMed ( $n=161)$; Science Direct $(n=74)$, Lilacs: $(n=16)$, Ebsco Host $(n=110)$, Google Académico $(n=515)$, TRIP Database $(n=12)$. Tabla II.

Después por los títulos y resúmenes a partir de los 888 artículos se excluyeron aquellos que no cumplieron con los criterios de inclusión, lo que dejó un grupo de 132 artículos. Luego de leer los resúmenes y someterlos a la valoración del contenido por parte del grupo de autores se obtuvieron 8 artículos para

Tabla II. Resultados de la búsqueda en bases de datos luego del tamizaje previo.

\begin{tabular}{lc}
\hline Base de datos consultada & Número de artículos \\
\hline Science Direct & 74 \\
PubMed & 161 \\
Lilacs & 16 \\
Ebsco Host & 110 \\
Google Académico & 515 \\
TRIP Database & 12 \\
\hline
\end{tabular}

lectura a texto completo. De estos, se excluyeron 6 artículos por las siguientes razones: Efectos de la expansión maxilar en sentido transversal sin dar respuesta a pregunta de investigación. Estudios que correlacionaban expansión transversal y apiñamiento en otro tipo de dentición. Artículos que, en su metodología, para obtener alineamiento de incisivos superiores combinaban expansión maxilar con aparatología fija ortodóntica (Tabla VII).

Finalmente, las características de los artículos se revisaron, se establecieron semejanzas y diferencias y se valoró su contenido (Tabla V). Los dos artículos seleccionados, resultaron con calidad moderada luego del análisis (Tabla VI). Esto implicó hacer una valoración cualitativa de los artículos para poder responder al objetivo de investigación.

La justificación de la Expansión maxilar, en ausencia de mordida cruzada posterior es aumentar el perímetro del arco maxilar y proporcionar espacio para corregir el apiñamiento de incisivos superiores en casos en los que el arco inferior se encuentra dentro del rango normal. La corrección del apiñamiento de incisivos superiores en dentición mixta, según algunos autores, es eficiente (Marco et al., 2016.

Tabla III. Escala de clasificación metodológica

\begin{tabular}{|c|c|c|}
\hline Temas de evaluación & & Puntaje \\
\hline 1. Diseño del estudio & & 9 puntos posibles \\
\hline \multicolumn{3}{|l|}{ A. Objetivo: El objetivo está claramente formulado } \\
\hline \multicolumn{3}{|l|}{ B. Población: Se describieron sus características } \\
\hline \multirow[t]{2}{*}{ C. Criterios de selección } & 1. Claramente descritos & \\
\hline & 2. Adecuados & \\
\hline \multirow[t]{2}{*}{ D. Tamaño de muestra } & 1. Adecuada & \\
\hline & $\begin{array}{l}\text { 2. Calculada antes de la recolección de los } \\
\text { datos }\end{array}$ & \\
\hline \multicolumn{3}{|l|}{$\begin{array}{l}\text { E. Características de línea base: Características similares de línea } \\
\text { base entre grupos de estudio }\end{array}$} \\
\hline \multicolumn{3}{|l|}{ F. Tiempo de medición: Prospectivo / Retrospectivo } \\
\hline \multicolumn{3}{|l|}{ G. Aleatorización: Establecida o descrita } \\
\hline 2. Medidas del estudio & & 5 puntos posibles \\
\hline \multicolumn{3}{|l|}{ H. Método de medición apropiado al obje tivo del estudio } \\
\hline \multirow[t]{2}{*}{ I. Mediciones a ciegas } & 1. Examinador & \\
\hline & 2. Estadístico & \\
\hline \multirow[t]{2}{*}{ J. Confiabilidad: calibración de examinadores } & 1. Descrita & \\
\hline & 2. Adecuado nivel de acuerdo & \\
\hline 3. Análisis estadístico & & 6 puntos posibles \\
\hline \multicolumn{3}{|l|}{ K. Medición de error: descrita } \\
\hline \multirow[t]{2}{*}{ L. Análisis estadístico } & 1. Apropiado para el tipo de dato & \\
\hline & 2. Análisis combinando de subgrupos & \\
\hline \multicolumn{3}{|l|}{ M. Factores de confusión: incluidos en análisis } \\
\hline \multirow[t]{2}{*}{ N. Nivel de significancia estadística } & Valor de $\mathrm{p}$ establecido & \\
\hline & Intervalos de confianza & \\
\hline
\end{tabular}


Tabla IV. Artículos analizados a texto completo y que fueron excluidos posteriormente.

\begin{tabular}{lll}
\hline \multicolumn{1}{c}{ Artículo } & \multicolumn{1}{c}{ Autores } & \multicolumn{1}{c}{ Motivo } \\
\hline $\begin{array}{l}\text { Sagittal and vertical effects of rapid maxillary } \\
\text { expansion in Class I, II, and III occlusions }\end{array}$ & $\begin{array}{l}\text { Farronato, G., Giannini, } \\
\text { L., Galbiati, G., }\end{array}$ & $\begin{array}{l}\text { Evalúa efectividad de la expansión maxilar } \\
\text { en maloclusiones Clase I, II y III. Pero no } \\
\text { para apiñamiento }\end{array}$ \\
$\begin{array}{ll}\text { Anchorage onto deciduous teeth: effectiveness } \\
\text { of early rapid maxillary expansion in increasing } \\
\text { dental arch dimension and improving anterior }\end{array}$ & $\begin{array}{l}\text { Mutinelli, S., Manfredi, } \\
\text { M., Guiducci, A., }\end{array}$ & $\begin{array}{l}\text { Correlaciona expansión transversal y } \\
\text { a piñami ento en edad temprana pero no en } \\
\text { dentición mixta. }\end{array}$
\end{tabular}
crowding

A prospective long-term study on the effects of rapid maxillary expansion in the early mixed dentition

Dental arch changes associated with rapid maxillary expansion: A retrospective model analysis study

Longitudinal clinical study on the effect of slow maxillary expansion with removable appliances

Influence of rapid palatal expansion on maxillary incisor alignment stability
Geran, R. G.,

McNamara, J. A.,

Baccetti, T., Franchi, L., Shapiro, L. M.

D'Souza, I. M., Kumar, Correlaciona expansión transversal y H., Shetty, K. S.

Moriyasu, K., Kuriyama, C., Kurihara, K., Fujihashi, A., Ohno, K., Asada, $Y$.

Canuto, L. F. G., de Freitas, M. R., Janson, G., de Freitas, K. M. S., Martins, P. P
Protocolo de expansión maxilar combinada con colocación de brackets en incisivos para corregir el apiñamiento.

a umento del perímetro de arco para aliviar el a piñami ento en el arco maxilar seguido de mecanoterapia fija.

Evalúa cambios en el arco dental en pacientes en dentición mixta tratados con expansión maxilar.

Compara estabilidad a largo plazo del alineamiento de incisivos en pacientes tratados con expansión rápida maxilar en dentición permanente

Tabla V. Descripción de los estudios seleccionados para el análisis final.

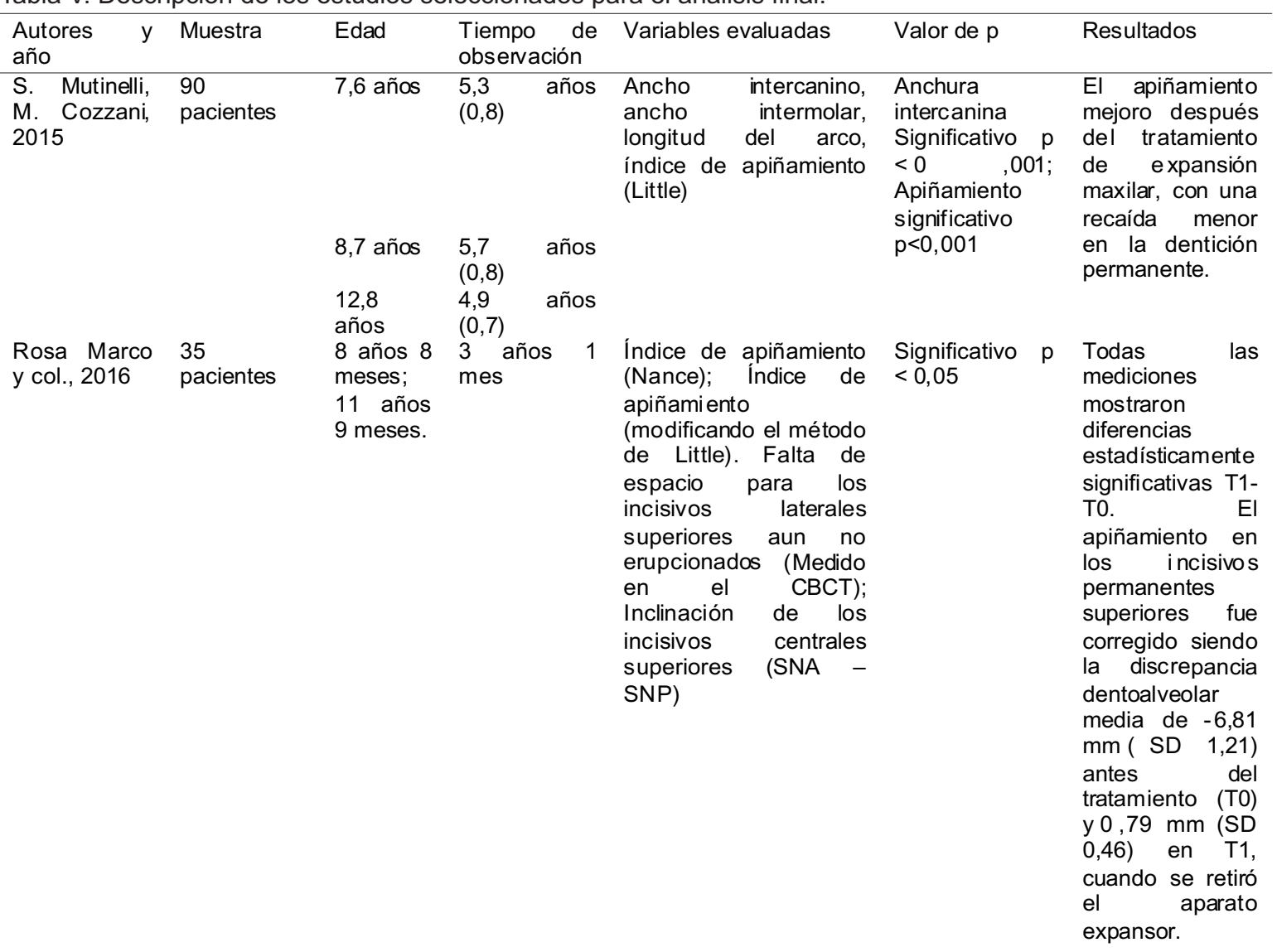


LONGLAX, T. M. C.; MONROY, G. J.; BOADA, C. N. J. \& LUGO, L. A. M. Efectividad de la expansión maxilar en la corrección de apiñamiento en dentición mixta. Revisión Sistemática. Int. J. Odontostomat., 14(1):101-108, 2020.

Tabla VI. Puntaje de calificación metodológica de los artículos seleccionados. Cumple totalmente el criterio (1 punto); cumple parcialmente el criterio (0,5 Puntos); no cumple el criterio (0 puntos).

\begin{tabular}{|c|c|c|c|c|c|c|c|c|c|c|c|c|c|c|c|c|c|c|c|c|c|c|}
\hline \multirow{2}{*}{ Autor/año } & \multirow{2}{*}{$\begin{array}{l}A \\
1\end{array}$} & \multirow{2}{*}{$\begin{array}{l}B \\
2\end{array}$} & \multicolumn{2}{|c|}{$\mathrm{C}$} & \multicolumn{2}{|c|}{$\mathrm{D}$} & \multirow{2}{*}{$\begin{array}{l}E \\
7\end{array}$} & \multirow{2}{*}{$\begin{array}{l}F \\
8\end{array}$} & \multirow{2}{*}{$\begin{array}{l}G \\
9\end{array}$} & \multirow{2}{*}{$\begin{array}{l}\mathrm{H} \\
10\end{array}$} & \multicolumn{2}{|c|}{1} & \multicolumn{2}{|c|}{$J$} & \multirow{2}{*}{$\begin{array}{l}\mathrm{K} \\
15\end{array}$} & \multicolumn{2}{|c|}{ I } & \multirow{2}{*}{$\begin{array}{l}M \\
18\end{array}$} & \multicolumn{2}{|l|}{$\mathrm{N}$} & \multirow{2}{*}{ Total } & \multirow[t]{2}{*}{ Calidad } \\
\hline & & & 3 & 4 & 5 & 6 & & & & & 11 & 12 & 13 & 14 & & 16 & 17 & & 19 & 20 & & \\
\hline Mutinelli et al., 2015 & 1 & 1 & 1 & 1 & 0 & 0 & 1 & 1 & 0 & 0 & 0 & 0 & 0 & 0 & 0 & 0 & 1 & 0 & 1 & 1 & 9 & Moderada \\
\hline $\begin{array}{c}\text { Rosa Marco et al., } \\
2016\end{array}$ & 1 & 1 & 1 & 1 & 0 & 0 & 0 & 1 & 0 & 0 & 0 & 0 & 1 & 0 & 0 & 1 & 1 & 1 & 1 & 1 & 11 & Moderada \\
\hline
\end{tabular}

\section{DISCUSIÓN}

En la presente revisión sistemática se incluyeron dos artículos, con un nivel de evidencia Moderada. Se encontró un artículo Retrospectivo y un artículo Prospectivo. En el artículo de Baccetti, se mostró evidencia de que los caninos impactados en dentición mixta se corrigen, con un protocolo de uso de expansión maxilar (Baccetti et al., 2009). Aunque el tratamiento de expansión maxilar aumenta la dimensión en el plano transversal del maxilar superior, resolviendo problemas de mordidas cruzadas principalmente; el uso de esta terapéutica en la corrección de caninos impactados genera el espacio para el adecuado posicionamiento y erupción de estos, en un plano vertical, lo cual se puede relacionar a la corrección de apiñamiento anterior por su cercanía a los caninos.

Sin embargo, en el estudio retrospectivo de Farranatoa, observó los efectos verticales y sagitales de la expansión maxilar en oclusiones clase I, II y III y determino cambios en la rotación del plano palatino hacia abajo y hacia atrás, con aumento en la altura facial total anterior y corrección de la hipoplasia maxilar, esto, dentro de los efectos verticales y sagitales, no encontró en sus resultados corrección de apiñamiento en incisivos superiores en dentición mixta (Farronatoa et al., 2011).

Igualmente, Moriyasu et al. ( 2010), en su estudio retrospectivo observó, que la utilización de esta terapia fue efectiva en el aumento del perímetro del arco, dando espacio así, para la erupción de caninos. Este espacio lo atribuyo, a la vestibularizacion de los demás dientes, siendo esta relación de espacio no estable en el tiempo. De este modo concluyó que la expansión maxilar es efectiva y estable en la corrección de deficiencias transversales del arco dental en la región molar.

Por otro lado, Mutinelli et al. reportaron (2015) que la expansión maxilar en el primer periodo transicional de la dentición es efectiva en el tratamiento de mordi- das cruzadas y determinó que el aumento de la longitud del arco puede mejorar el alineamiento de los dientes superiores anteriores que presenten un apiñamiento moderado (Mutinelli et al., 2006); pero, contradice la teoría anterior de Geran et al., al reportar, que la terapia de expansión en dentición mixta puede considerarse efectiva como tratamiento para corregir deficiencias transversales del maxilar, y consigue la alineación al colocar brackets en los incisivos superiores (Geran et al.).

Al igual que D'Souza y Shetty, cuando evaluaron los cambios en el arco dental provocados por la expansión maxilar seguido de tratamiento ortodóntico fijo (D'Souza et al., 2015). Lograron corregir el apiñamiento combinando expansión maxilar y ortodoncia con aparatología fija. La investigación concluye que la expansión del arco puede aumentar significativamente el perímetro, ayudando al clínico a tratar casos con no extracciones. Canuto et al., en su estudio retrospectivo, determinaron, la estabilidad a largo plazo del alineamiento de los incisivos superiores, comparando pacientes con y sin extracciones, tratados con expansión rápida y tratamiento de ortodoncia (Canuto et al.). Concluyeron que la estabilidad fue similar en los dos grupos, y que la expansión maxilar no influencia la estabilidad del alineamiento a largo plazo.

Mutinelli \& Cozzani, proporcionaron evidencia, al demostrar que el apiñamiento, en un nivel bajo, mejora espontáneamente, utilizando como tratamiento la expansión maxilar en dentición mixta; refiere que en cuanto mejor sea el alineamiento previo al tratamiento en dentición permanente, mejor será la estabilidad a largo plazo. Supone, que el aumento en la longitud del arco antes de la erupción de los incisivos laterales superiores permite que estos dientes hagan erupción en una posición más recta. Las fibras transeptales del ligamento periodontal, pueden penetrar las raíces de dientes mejor alineados, con la consiguiente estabilidad a largo plazo de la alineación anterior maxilar (Mutinelli \& Cozanni). 
Finalmente, Marco et al., en su estudio clínico prospectivo en el que incluyeron a 35 pacientes, tratados por el mismo ortodoncista, que presentaban apiñamiento de los incisivos permanentes superiores en la dentición mixta temprana en ausencia de mordida cruzada posterior, obtuvieron como resultado al instaurar en los pacientes un protocolo de expansión maxilar que alivió el apiñamiento de incisivos en todos ellos. La expansión maxilar ortopédico temprana, parece ser una anticipación del crecimiento maxilar transversal, que normalmente se producirá en un período de tiempo más largo, proporcionando espacio para corregir el apiñamiento (Marco, Patrizia et al.). Según los autores, el protocolo se debe realizar anclando el aparato en molares deciduos, ya que, si se ancla a molares permanentes, y no hay presencia de mordida cruzada, puede producir una mordida en tijera posterior y un trastorno oclusal. El mejor momento es durante la dentición mixta, justo antes de la erupción de los incisivos laterales permanentes superiores y después de que los molares permanentes estén completamente erupcionados y acoplados en oclusión. El alivio interceptivo del apiñamiento de incisivos superiores en la dentición mixta temprana con Expansión Rápida Palatina, anclado a dientes deciduos en ausencia de una mordida cruzada posterior es aparentemente una modalidad de tratamiento eficiente.

\section{CONCLUSIONES}

En relación a los resultados de la presente Revisión sistemática se concluye que existen pocos estudios que relacionen la técnica de expansión maxilar con la corrección de apiñamiento en dentición mixta. Adicionalmente, existe evidencia moderada frente al hecho de que la expansión maxilar corrige el apiñamiento en dentición mixta. Los estudios que reportan el éxito de esta terapia en la resolución del apiñamiento no tienen la suficiente calidad metodológica para llevar a una toma de decisión. Esto quiere decir que se requiere más investigación que les permita a los clínicos establecer evidencia adecuada para determinar si la aplicación de la expansión maxilar permite la corrección del apiñamiento.

LONGLAX, T. M. C.; MONROY, G. J.; BOADA, C. N. J. \& LUGO, L. A. M. Effectiveness of the maxillary expansion in the correction of crowding and mixed dentition. Systematic review. Int. J. Odontostomat., 14(1):101-106, 2020.
ABSTRACT: Crowding at early ages is reflected when tooth size does not coincide with arch length. Thus, it is important to determine whether maxillary expansion alone could solve the problem of crowding in the anterior sector, bearing in mind that this treatment is one of the oldest and has commonly focused on solving transverse alterations in posterior teeth used at an early age. The objective was to determine whether or not maxillary expansion is effective in correcting crowding in the mixed dentition by analysing the available evidence. A systematic review was carried out in Spanish and English, to establish the maximum possible evidence, with the indications of the PRISMA guide. Two articles met the quality of the review. The first established that crowding improved after the treatment of maxillary expansion, with a minor recurrence in the permanent dentition. In the second, it was found that crowding in the upper permanent incisors was corrected when the expanding appliance was removed. The current evidence is not of sufficient methodological quality to lead to a decision. Randomized clinical trials are recommended in this area.

KEY WORDS: mixed dentition, preventive orthodontics, malocclusion, revision (DeCS).

\section{REFERENCIAS BIBLIOGRÁFICAS}

Koo , T. \& Li, M. A Guideline of Selecting and Reporting Intraclass Correlation Coefficients for Reliability Research. J. Chiropr. Med., 15(2):155-63, 2016.

van der Linden, F. Myths and Legends in Orthodontics. Eur. J. Orthod., 2008.

Arif, A.; Rasheed, T. \& Ali, A. Dental crowding and its relationship to tooth size and arch dimensions. J. Nat. Scie. Res., 14(10):1336, 2014.

Baccetti , T.; Leonardi , M. \& Armi, P. A randomized clinical study of two interceptive approaches to palatally displaced canines. Eur. J. Orthod., 30:381-5, 2008.

Baccetti, T., Mucedero, M., Leonardi, M., \& Cozzad, P. Interceptive treatment of palatal impaction of maxillary canines with rapid maxillary expansion: a randomized clinical trial. Am. J. Orthod. Dentofacial Orthop., 136(5):657-61, 2009.

Barber Starr, P. C. Malocclusion: How important is that bite? J. Pediat. Health Care, 13(5):245-7, 1999.

Bartzela, T. \& Jonas, I. Long-term stability of unilateral posterior crossbite correction. Angle Orthod., 77(2):237-43, 2007.

Bragge, P. Asking good clinical research questions and choosing the right study design. Int. J. Care Injured, 41S:S3-S6, 2010.

Canuto, L.; de Freitas , M.; Janson, G.; de Freitas, K. \& Martins, P. Influence of rapid palatal expansión on maxillary incisor alignment stability. Am. J. Orthod. Dentofacial Orthop., 137(2):164.e1164.e6, 2010.

Cozzani, M.; Guiducci, A.; Mirenghi, S.; Mutinelli, S. \& Siciliani , G. Arch width Changes with a Rapid Maxillary Expansion Appliance Anchored to the Primary Teeth. Angle Orthod., 77(2):296-302, 2007.

Doris, J.; Bernard, B.; Kuftinec , M. \& Stom, D. A Biometric study of tooth size and dental crowding. Am. J. Orthod., 79:326-36, 1981.

D'Souza, I.; Kumar , H. \& Shetty, K. Dental arch changes associated with rapid maxillary expansión: A Retrospective model analysis study. Contemp. Clin. Dent., 6(1):51-7, 2015. 
Farronatoa, G.; Gianninib, L.; Galb, G. \& Cinzia Maspero. Sagittal and vertical effects of rapid maxillary expansión in Class I, II and III occlusions. Angle Orthod., 81(2):298-303, 2011.

Geran, R.; McNamara, J.; Baccetti, T.; Franchi, L. \& Shapiro, L. A prospective long-term study on the effects of rapid maxillary expansión in the early mixed dentition. Am. J. Orthod. Dentofacial Orthop., 129(5):631-40, 2006.

Graber Thomas M, V. R. Ortodoncia Principios y técnicas actuales. 4 ed. Madrid, Elsevier, 2006.

King, G. J. \& Brudvuk, P. Effectiveness of interceptive orthodontic treatment in reducing malocclusions. Am. J. Orthod. Dentofacial Orthop., 137(1):18-25, 2010.

Howe, R.; McNamara, J. \& O'Connor, K. An examination of dental crowding and its relationship to tooth size and arch dimension. Am. J. Orthod., 85(5):363-73, 1983.

Johansen, M. \& Thomsen, S. Guidelines for Reporting Medical Research: A Critical Appraisal. Int. Sch. Res. Not., 2016. 1346026, 2016.

Kau, C. H, D. P. Extractions as a form of interception in the developing dentition: a randomized controlled trial. J. Orthod., 31:107-14, 2004.

Lagravere, M.; Major, P. \& Flores-Mir, C. Long term skeletal change with rapid maxillary expansion: a systematic review. Angle Orthod., 75(6):1046-52, 2005.

Liberati, A.; Altman, D.; Tetzlaff, J.; Mulrow, C.; Gøtzsche, P.; loannidis, J. \& Moher, D. The PRISMA Statement for Reporting Systematic Reviews and Meta-Analyses of Studies That Evaluate Health Care Interventions: Explanation and Elaboration. PLOS Med., 6(7):e1000100, 2009.

Lione, R.; Franchi, L. \& Cozza, P. Does rapid maxillary expansion induce adverse effects in growing subjects? Angle Orthod., 83(1): 172-82, 2013

Lombardi, A. The adaptive value of dental crowding: A consideration of the biologic basis of malocclusion. Am. J. Orthod., 81(1): 3842, 1982

Lundstrom, A. The aetiology of crowding of the teeth (based on studies of twins and morphological investigations) and orthodontic treatment (expansión or extraction). Eur. Orthod. Soc., 21(176): 176-91, 1951.

Mafla, B. D. Maloclusión y necesidad de tratamiento ortodóntico en adolescentes de Pasto, Colombia. Rev. Fac. Odontol. Univ. Antioq., 22(2):173-85, 2011.

Marco, R.; Patrizia, L.; Manti, G. \& Caprioglio, A. Rapid Palatal expansión in the absence of posterior cross-bite to intercept maxillary incisor crowding in the mixed dentition: a CBCT evaluation of spontaneous changes of untouched permanent molars. Paediatr. Dent., 17(4), 2016.

Marshall, S.; English, J.; Huang, G.; Messersmith , M.; Nah, H.; Riolo, M. \& Turpin, D. Long-term stability of maxillary expansión. Am. J. Orthop, 133(6):780-1, 2008.

McKeown, M. The diagnosis of incipient arch crowding in children. N. Z. Dent. J., 349:93-6, 1981.

Moriyasu, K.; Kuriyama, C.; Kurihara, K.; Fujihashi, A.; Ohno, K. \& Asada, Y. Longitudinal clinical study on the effect of slow maxillary expansión with removable appliances. Pediat. Dent. J. 20(1): 78-83, 2010.

Mutinelli, S. \& Cozanni, M. Rapid maxillary expansión in early mixed dentition: effectiveness of increasing arch dimensión with anchorage on deciduous teeth. Eur. J. Paediat. Dent., 16(2):11522, 2015

Mutinelli, S.; Manfredi, M.; Guiducci, A.; Denotti, G. \& Cozzani, M. Anchorage onto deciduous teeth: effectiveness of early rapid maxillary expansión in increasing dental arch dimensión and improving anterior crowding. Prog. Orthod, 16(22):7, 2015.

Normando, D.; Almeida, M. \& Quintão, C. Dental crowding: the role of genetics and tooth wear. Angle Orthod., 83(1):10-5, 2013.
Radnzic, D. Dental crowding and its relationship to mesiodistal crown diameters and arch dimensions. Am. J. Orthod. Dentofacial Orthop., 94:50-6, 1988.

Sunnak, R.; Johal, A. \& Fleming, P. Is orthodontics prior to 11 years of age evidence - based? A systematic review and meta analysis. J. Dent., 43(5):477-86, 2015.

Trevethan, R. Intraclass correlation coefficients: clearing the air, extending some cautions, and making some requests. Health Serv. Outcomes Res. Method., 17:127-43, 2017.

Vaughn GA, M. B. The effects of maxillary protraction therapy with or without rapid palatal expansion: a prospective, randomized clinical trial. Am. J. Orthod. Dentofacial Orthop., 128:299-309, 2005.

Warren, J. J. B. S. Tooth size-arch length relation ships in the deciduous dentition: a comparision between contemporary and historical samples. Am. J. Orthod., 123(6):614-9, 2003.

Tang, Z.; Jiang, L.P. \& Wu, J.Y. Effect of maxillary expansion on orthodontics. Asian Pacific J. Tropical Medi., 8(11):944-51, 2015.

Direccción para correspondencia:

Gustavo Jaimes Monroy

Universidad Antonio Nariño

Facultad de Odontología

Bogotá

COLOMBIA

Email: tavojaimes@uan.edu.co

Recibido : 07-06-2019

Aceptado: 25-07-2019 